\title{
Povidone-iodine for the management of oral mucositis during cancer therapy
}

\author{
Jeeve Kanagalingam, ${ }^{1}$ Akhil Chopra, ${ }^{2}$ Min Hee Hong, ${ }^{3}$ Wisam Ibrahim, ${ }^{4}$ Antonio Villalon, ${ }^{5}$ \\ Jin-Ching Lin 6
}

${ }^{1}$ Lee Kong Chian School of Medicine, Johns Hopkins Singapore IMC and Tan Tock Seng Hospital, The ENT Clinic, Mount Elizabeth Novena Hospital, Singapore; ${ }^{2}$ OncoCare, Mount Elizabeth Medical Centre, Mount Elizabeth, Singapore; ${ }^{3}$ Division of Medical Oncology, Department of Internal Medicine, Yonsei Cancer Center, Severance Hospital, Yonsei University Health System, Seoul, Korea ${ }^{4}$ Ibn Nafees Medical Center, Abu Dhabi, United Arab Emirates; ${ }^{5}$ Manila Doctors Hospital, Manila, Philippines; ${ }^{6}$ Department of Radiation Oncology, Taichung Veterans General Hospital, Taichung, Taiwan

\begin{abstract}
Oral mucositis (OM) is a common and often dose-limiting side effect of cancer therapy. Povidone iodine (PVP-I) formulations have been shown to decrease the incidence and severity of $\mathrm{OM}$, but the relevance of these findings remains unclear. The objective of the present study was to review evidence for the use of PVP-I for OM management. An algorithm identified relevant articles published online, and a panel of experts with experience in the management of OM reviewed the findings. Six studies fulfilled the criteria for full review. Two studies provided evidence of moderate quality. Two of the studies with negative findings were confounded by the use of PVP-I concentrations that are too low to be efficacious. The remaining two studies were found to have design flaws. There exists reasonable evidence to support a recommendation for the use of PVP-I in the management of cancer therapy-related OM.
\end{abstract}

Correspondence: Jin-Ching Lin,

Department of Radiation Oncology,

Taichung Veterans General Hospital, Taichung, Taiwan.

E-mail: jclin@vghtc.gov.tw

Key words: Povidone iodine; chemotherapy; radiotherapy; cancer; mucositis.

Funding: this study was funded by Mundipharma Pte Ltd, Singapore.

Acknowledgements: editing services were provided by Streamline Editing Korea.

Received for publication: 26 April 2017.

Revision received: 9 August 2017.

Accepted for publication: 21 August 2017

This work is licensed under a Creative Commons Attribution NonCommercial 4.0 License (CC BY-NC 4.0).

(C) Copyright J. Kanagalingam et al., 2017

Licensee PAGEPress, Italy

Oncology Reviews 2017; 11:341

doi:10.4081/oncol.2017.341

\section{Introduction}

Oral mucositis (OM) is considered one of the most severe non-haematological complications of cancer therapy, affecting more than $40 \%$ of patients undergoing some types of chemotherapy and almost all head and neck cancer patients undergoing radiotherapy. ${ }^{1,2}$ The condition is characterized as an inflammatory process that disrupts the mucous lining of the oral cavity and parts of the pharynx. Symptoms include oedema, ulceration and bleeding, with patients often having difficulty swallowing or talking. The associated ulcers and oral lesions that develop can cause significant pain, to the extent where patients may be unable to eat solid food or even ingest liquids at severe stages of OM. In extreme cases, a more serious form called confluent mucositis can arise, lining the patient's mouth and tongue with a white mucus coating. OM is thought to occur independently of oral mucosal infections of viral and fungal aetiology, but it may be exacerbated by such concomitant infections. Therefore, good oral hygiene practices are emphasized to prevent lesions becoming infected with pathogenic microbial flora, of which immunosuppressed patients are likely to be at greater risk. ${ }^{3}$

Severe OM in patients is a dose-limiting toxicity of cancer therapy as it can necessitate physicians reducing or omitting planned interventions. ${ }^{4}$ In very severe cases, treatment interventions are interrupted and patients may require hospitalization. 5,6 Such dosage modifications or the discontinuation of recommended chemo/radiotherapy regimens can have a negative impact on clinical outcomes. The development of effective regimens for severe $\mathrm{OM}$ is therefore necessary to improve quality of life and treatment outcomes for cancer patients.

Due to heterogeneity in study designs relating to differing aims, methodology and design quality, the evidence available is conflicting and confusing. ${ }^{7,8}$ The Journal of the National Comprehensive Cancer Network (NCCN) published the NCCN Task Force Report: Prevention and Management of Mucositis in Cancer Care in 2008, which makes suggestions for good practice but due to the paucity of strong evidence does not make any recommendations for the treatment of OM. ${ }^{2}$

In order to provide clearer guidance for clinicians, the Multinational Association of Supportive Care in Cancer (MASCC) in collaboration with the International Society of Oral Oncology (ISOO) conducted a comprehensive assessment of the literature to develop evidence-based and consensus-backed OM clinical practice guidelines. ${ }^{9}$ The MASCC/ISOO guidelines are based on evidence from studies looking at interventions used in 
specific cancer treatment regimens. ${ }^{10}$ For example, benzydamine is only recommended for head and neck cancer patients receiving moderate dose radiotherapy (up to 50 Gy) without concomitant chemotherapy. This is clinically relevant only to a minority of head and neck cancer patients. However, at least some of the suggested therapeutic agents may not be available in all markets or affordable for all patients. A more universal approach to OM management therefore requires the additional consideration of regulatory and economic factors at play.

While a number of antimicrobial mouthwashes are available, iodophore-based formulations including povidone iodine (PVP-I) have remained popular as antimicrobial agents. PVP-I formulations have a long track record of safe and effective use for the treatment of antisepsis and wound healing. ${ }^{11}$ The iodine within PVP-I forms a complex with the synthetic carrier polymer polyvinyl pyrrolidine, and in an aqueous medium, free iodine is released. PVP-I complexes release free iodine into the medium and an equilibrium is established, with further free iodine molecules released from PVP-I as iodine-consuming germicidal activity proceeds. This concentration-dependent equilibrium of free iodine to PVPbound iodine helps to address tolerability issues. ${ }^{12}$ The anti-microbial properties associated with iodine involve the inhibition of critical cellular mechanisms via the oxidation of nucleotides, amino acids and fatty acids in cell membranes. ${ }^{13}$ PVP-I has been shown to be effective against both gram-negative and gram-positive bacteria, yeasts, protozoa and viruses. ${ }^{14}$ An additional feature of relevance is that there have been no documented cases of bacterial resistance to iodine, likely due to its multiple mechanisms of action. ${ }^{15}$ In vitro evidence suggests that iodine not only has broadspectrum antibacterial, antifungal and antiviral effects, but also anti-inflammatory effects that have been proven to inhibit inflammation arising from the host response. ${ }^{16}$ These effects have been shown to be clinically relevant in several conditions. ${ }^{17}$ For these reasons, PVP-I formulations are extensively used for surgical site preparation, wound management, and feminine care.

Although randomized clinical trials using PVP-I oral gargle for the treatment of OM have been published, the overall implications of these findings have not been thoroughly addressed. Both positive and negative data for the use of PVP-I has been found in the literature. The 2014 MASCC/ISOO guidelines note that studies of PVP-I oral gargle present inadequate or conflicting data, concluding with the designation No guideline possible. ${ }^{10}$ However, concerns have been raised that at least some of the negative findings reported in the literature may be misleading, as a number of studies have used final concentrations of oral PVP-I that are well below the recommended effective concentration. The current review was undertaken to systematically assess the overall level of evidence present in the literature regarding PVP-I in the treatment of OM.

\section{Materials and Methods}

\section{Literature search}

A literature search was conducted to identify clinical OM studies of satisfactory relevance. Expert panellists were responsible for reviewing the studies and providing feedback. These panellists are all listed as co-authors of this report and were selected based on their experience in managing OM in a multidisciplinary setting. The method for literature review and assessment was agreed with the authors at the beginning. We excluded review/systematic review articles, reports from duplicated publications or follow-up reports from the same study, clinical practice/chart reviews, in vitro/in vivo non-clinical studies, and interventions with povidone iodine as a component of combination treatment, rather than as a single agent (Table 1 and Figure 1).

\section{Inclusion/exclusion criteria}

Inclusion criteria were English language publications reporting testing of a PVP-I intervention for OM in humans in relation to cancer, published in a peer-reviewed journal, and indexed in Medline on or before August 31, 2016. Aspects such as selection

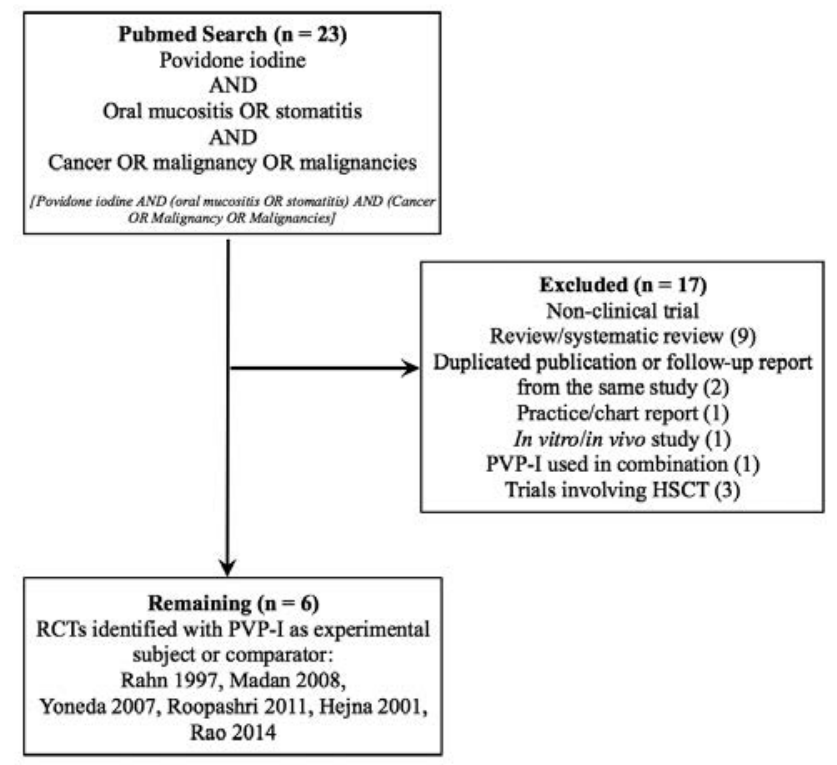

Figure 1. Flow diagram of search results. HSCT, hematopoietic stem cell transplantation; RCT, randomized controlled trials.

Table 1. Inclusion and exclusion criteria for literature search.

\begin{tabular}{ll} 
Inclusion & Exclusion \\
Povidone iodine (PubMed term) & Non-clinical trial \\
Oral mucositis OR stomatitis (PubMed term) & Review/systematic review \\
\hline Cancer OR malignancy OR malignancies (PubMed term) & Duplicated publication or follow-up report from the same study \\
Published in peer-reviewed journal (online-only publications permitted for inclusion) & Practice/chart report \\
\hline All age groups & In vitro/in vivo non-clinical study \\
& Povidone-iodine as part of combination, rather than single agent \\
& Trials involving haematopoietic stem cell transplantation \\
\hline
\end{tabular}


of patients, allocation of patients to treatment groups, therapeutic regimen, study administration, withdrawals from study, patient blinding (randomized controlled trials only), measurements and statistical analysis were evaluated. Upon review of the articles presented, 6 expert panellists (the authors of this review) with extensive experience in the treatment of $\mathrm{OM}$ provided their evaluation of: i) the level (quality) of the evidence in the studies supporting PVP-I usage for the treatment of OM; and ii) the class (strength) of recommendation for its usage in OM management (see Addendum for recommendation criteria).

\section{Results}

Of the 23 abstracts initially identified in the Medline search, 6 were selected for in-depth review in accordance with the selection algorithm. The studies are summarized in Table 2.

Rahn et al. investigated the efficacy of PVP-I for the prophylaxis of mucositis during antineoplastic radiochemotherapy. ${ }^{18}$ Forty patients were randomly assigned to a treatment or control group, with all receiving prophylaxis of mucositis with nystatin, dexpanthenol, rutoside and immunoglobulin. In addition, the patients of the treatment group performed rinsing 4 times daily with $1 \%$ PVP-I, and the control patients with sterile water. The onset of OM in the control group was at 1.5 weeks from the start of cancer therapy as opposed to 2.25 weeks in the treatment group. The mean total duration of mucositis was 2.75 weeks in patients receiving PVP-I treatment and 9.25 weeks in control patients $(\mathrm{P}<0.001)$, with mean area under the curve (AUC) values of 2.5 in treatment patients versus 15.75 for the control patients $(\mathrm{P}<0.001)$. Although this study is now somewhat dated as prophylaxis with nystatin is no longer considered standard, the differences observed in this trial were both statistically and clinically significant. It was concluded that rinsing with PVP-I reduced the incidence, time to onset, severity and duration of oral mucositis during antineoplastic radiochemotherapy.

Similarly, in a trial by Madan et al., 80 patients with head and neck cancer undergoing curative radiotherapy were treated with either $0.12 \%$ chlorhexidine, $1 \%$ PVP-I or salt/soda mouthwashes. ${ }^{19}$ It was found that patients receiving PVP-I had significantly lower OM scores compared to both the chlorhexidine and salt/soda groups after 5 weeks of treatment. It was therefore concluded that PVP-I could reduce the severity and delay the onset of cancer therapy-related OM.

Yoneda et al. found that a $0.5 \%$ solution of PVP-I when included as part of an oral hygiene rinse program using a special electronic dental e-brush with irrigation and suctioning of the mouth significantly lowered the incidence of OM. ${ }^{20}$ In addition, the proportion of subjects with newly detected opportunistic pathogens was higher in the non-special care group, for which the authors concluded that irrigation with PVP-I is likely to improve patient quality of life and may reduce hospitalization times. Although the study concluded that PVP-I is effective for the treatment of OM, it is important to note that unlike the other five studies, this study used patients with esophageal cancer (which may not always be technically classified as a head and neck cancer), which diversifies the patient user group.

A study by Roopashri et al. compared benzydamine (0.15\%), chlorhexidine (0.2\%) and PVP-I (5\%), and found evidence of efficacy for all interventions, ${ }^{21}$ but concluded that benzydamine was the superior agent. In fact, the incidence of mucositis was not statistically different in the study and control groups. The observed differences in severity of mucositis and pain did not meet statistical significance. However, the authors did not adequately describe the overall trial design, particularly in relation to blinding. Despite stating that the mouth-rinsing regimen was under professional supervision, it was concluded that compliance with benzydamine was superior. The impact of the findings and the extent of benefit afforded by PVP-I or other treatments in this study is therefore difficult to discern.

The study design employed by Hejna et al. tested PVP-I together with amphotericin B (antiseptic agent group) as the comparator for GM-CSF in the prevention and treatment of OM. ${ }^{22}$ The author concluded that GM-CSF treatment resulted in delayed onset and shorter duration of OM. It is noteworthy that the final concentration of PVP-I oral gargle - though not explicitly stated - is significantly lower than the current recommended concentration of at least $1 \%$. The investigators state $4 \mathrm{~mL}$ of betaisodina (7.5\% PVPI) was diluted in $125 \mathrm{~mL}$ of water, implying a final concentration of $0.24 \%$. This concentration represents a less than 1 in 4 dilution of the recommended strength.

Rao et al. evaluated the efficacy of turmeric in preventing radiation-induced mucositis. ${ }^{23}$ In a single-blinded, randomized, controlled clinical trial conducted with head and neck cancer patients requiring 70 Gy of radiation or chemoradiotherapy, patients were randomly assigned to receive either turmeric gargle or PVP-I as an active comparator. The study concluded that gargling with turmeric by head and neck cancer patients undergoing radiation therapy was superior to PVP-I in delaying and reducing the severity of mucositis. PVP-I was used as a comparator at a final concentration approximately 10 -fold below that which is recommended. It is therefore likely that the absence of efficacy for PVP-I in this study was entirely due to its low concentration.

In summary, the 3 studies conducted by Rahn, Madan and Yoneda found evidence for the efficacy of PVP-I in alleviating the symptoms of OM. In contrast, the studies by Roopashri, Hejna and Rao did not support PVP-I usage. However, the study by Roopashri suffers from a lack of statistically significant findings, whilst the Hejna and Rao studies used very low concentrations of PVP-I that explain the lack of efficacy.

When the expert panelists who reviewed the studies were asked for their evaluation of the overall level (quality) of evidence considering the strengths of each study, five responded that there was moderate-quality evidence from one or more randomized controlled trials, while the remaining panelist indicated that there was moderate-quality evidence from one or more well-designed, wellexecuted nonrandomized studies, observational studies, or registry studies. Similarly, when asked on the level of recommendation warranted due to this evidence, one panelist gave a recommendation of strong, while the remaining five panelists provided a recommendation of moderate (taking into account the risk/benefit profile). The slight discrepancy in opinion may have been due to the different clinical settings and standards familiar to the different study coauthors (who were all from different countries).

\section{Discussion}

The overall objective of the present study was to provide an objective review of the literature in regards to the use of PVP-I for the management of OM. We sought to critically evaluate 6 of the most relevant clinical trials in the literature that used PVP-I gargle in the treatment or prevention of cancer-related OM, of which the Rahn, Madan, and Yoneda studies were determined as having positive evidence. The Hejna and Rao studies showed negative results, however, this was thought likely to be due to the stated PVP-I concentrations being several-fold below that which is recommended. The study by Roopashri reported only descriptive terms for its 


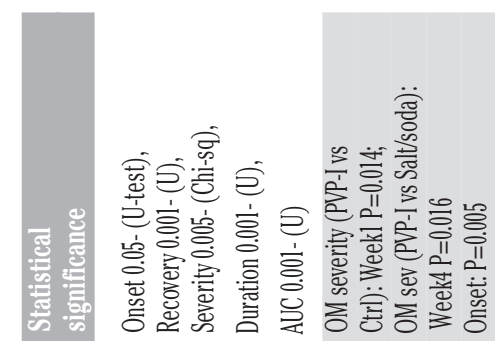

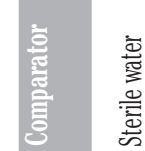

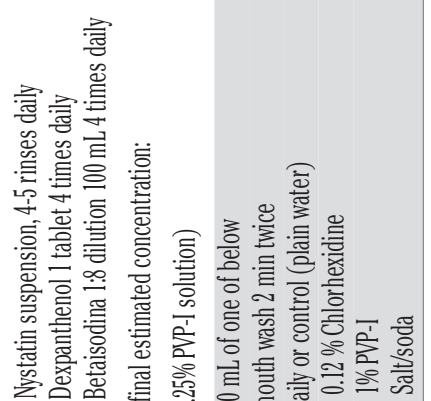

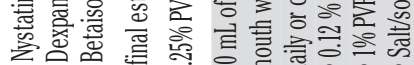

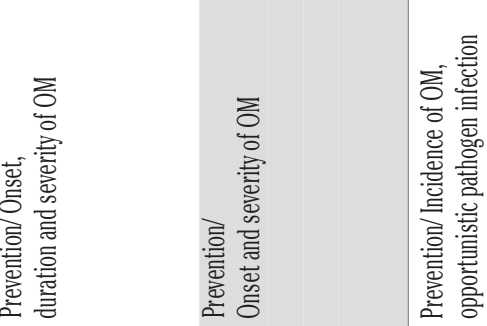

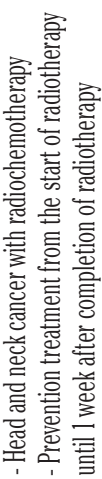

产

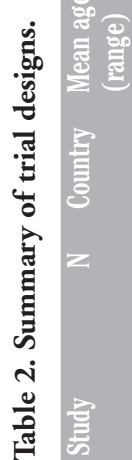

产

龸棺

衰

우

品

高

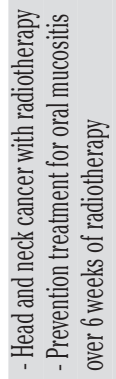

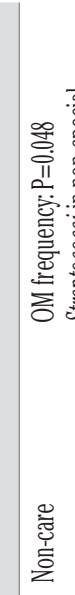

을

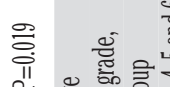

ت

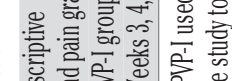
这

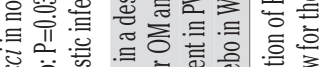

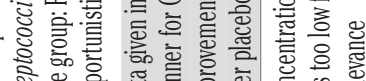

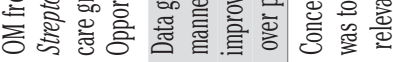
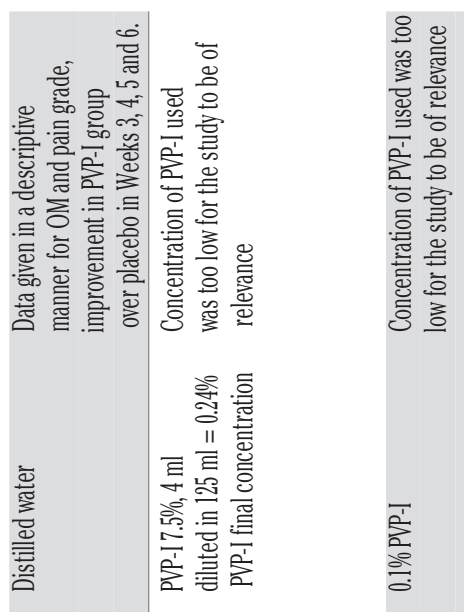

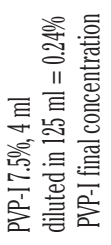

$\frac{\bar{c}}{\frac{1}{2}}$

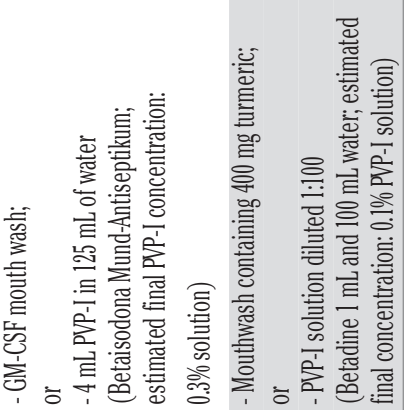

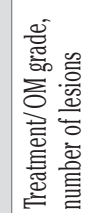

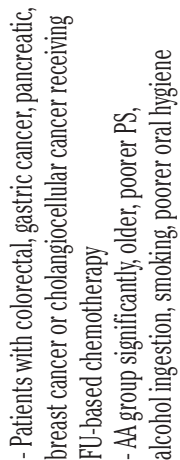

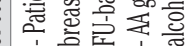

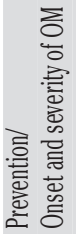
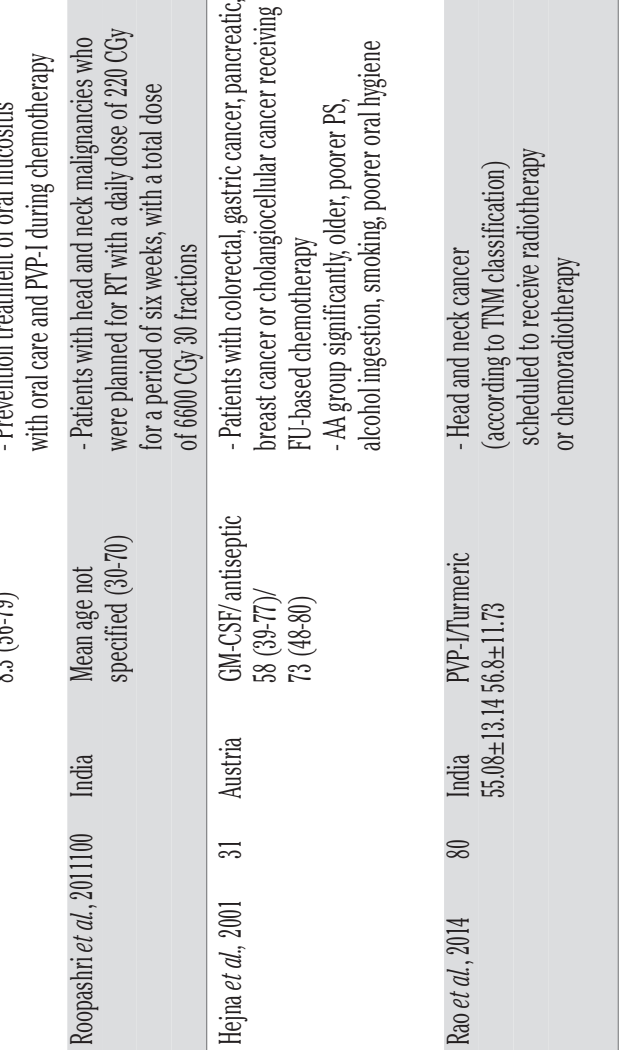

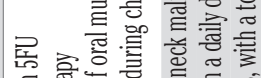

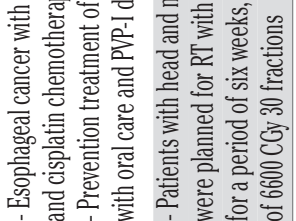

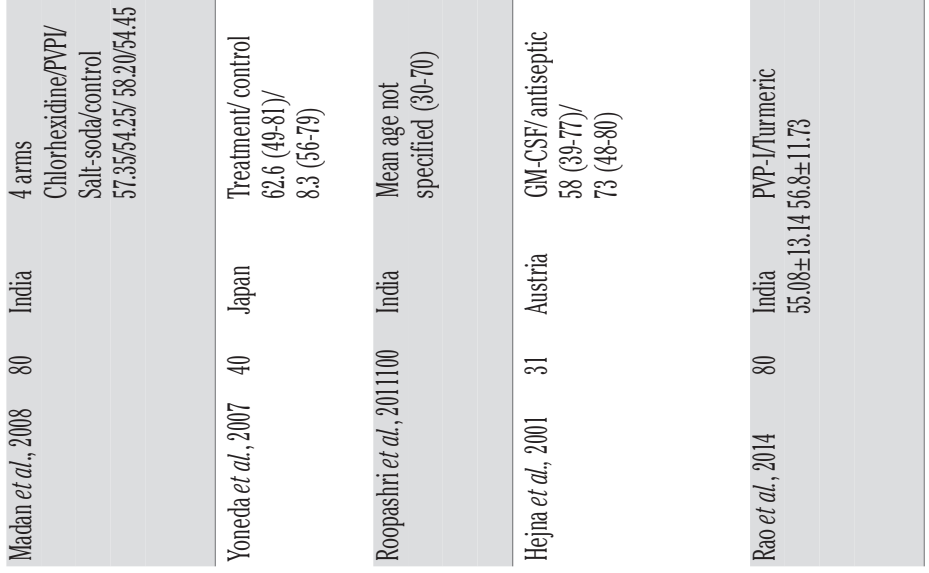


results, with no statistically significant conclusions. PVP-I gargle is marketed for oral care at $1 \%$ concentration, and it appears that a common flaw in methodology is its inappropriate dilution during clinical application to levels that would be non-efficacious. In contrast, for the randomized studies with concentrations ranging between $0.5-1 \%$ PVP-I, all three showed the effective alleviation of OM-related symptoms. The differing conclusions of the studies may be due to differences in the tumor types, patient populations and chemo/radiotherapy regimens used. These differences were not always discernable. For example, the Yoneda study listed the 5-FU regimen used but not the dosage of radiotherapy, while the Hejna study simply stated, patients who had undergone radiotherapy of head and neck were eligible, if at least 6 weeks had elapsed since termination of therapy and the resolution of acute toxic effects of treatment. In contrast, the Madan and Rao studies used identical radiation treatment regimens ( 2 Gy per day, five times a week without any intended gaps for a planned target dose of 70 Gy (7 consecutive weeks). It is recommendable for future reports to list the full details of chemo/radiotherapy regimens used in order to better determine the causes for distinct findings.

The oral mucosa has a rapid rate of cellular turnover, and is therefore highly susceptible to cancer treatment-related toxicity caused by chemotherapy and ionizing radiation. ${ }^{24}$ Complications with inflamed tissue can also arise due to the diverse and complex microflora that colonizes the oral cavity. The effective treatment of $\mathrm{OM}$ therefore likely requires both the inflammatory and microbial aspects to be addressed. ${ }^{25}$ As an agent that exhibits potent and clinically relevant anti-inflammatory and antimicrobial properties, ${ }^{14,17}$ PVP-I formulations could therefore impact the pathophysiological mechanisms responsible for OM.

Strong mechanistic rationale exists for the application of PVPI in treating the symptoms of OM. In vitro and in vivo studies have revealed potent anti-inflammatory, antiseptic, anti-oedematous, and anti-hemostatic effects in a wide range of models. The antiinflammatory effects of PVP-I on microbial-induced cytokine generation have been observed in various cell lines, including human neutrophils. TNF- $\alpha$ is an important mediator of inflammation and its dysregulation is implicated in various chronic diseases. It was demonstrated that PVP-I suppresses TNF- $\alpha$ release from human neutrophils in response to stimulation by Staphylococcus aureus and Respiratory Syncytial Virus epitopes. Similarly, the enzyme Bgalactosidase is considered a marker of bacterial activity during the inflammatory process. Treatment with varying concentrations of PVP-I has been shown to reduce $\beta$-galactosidase activity in Escherichia coli cultures and supernatant. ${ }^{26}$

A position paper released by the European Wound Management Association (EWMA) notes the broad spectrum of PVP-I activity against both gram positive and negative bacteria, fungi, endospores and viruses. ${ }^{26}$ While evidence of cross-resistance to antiseptics and antibiotics has been documented for many agents, acquired resistance and cross-resistance has been rarely reported for iodine when used for specific indications. ${ }^{27}$ This lack of resistance is thought to be due to the diverse mechanisms through which iodine exerts its effects.

PVP-I is also likely to have anti-oedematous effects. A singleblind randomized study was conducted to evaluate facial swelling in postoperative patients over varying time intervals. In the treatment group, a $0.5 \%$ PVP-I solution at a concentration of 0.5 $\mathrm{mg} / \mathrm{mL}$ caused a significant reduction in postoperative swelling compared to the control group $(\mathrm{P}<0.01)$. This effect of PVP-I was suggested to be due to its inhibitory effect on leukotriene B4 and leukocyte extravasation. ${ }^{28}$

Further investigation into the anti-hemostatic effects of PVP-I have similarly revealed important findings. In a study of fresh bleeding following irrigation of the extraction socket in patients that have had a tooth removed, 19 of 25 patients in the treatment group showed spontaneous cessation of bleeding upon application of PVP-I, which was observed in only 5 of the 25 patients irrigated with saline. These observations were made prior to drying the socket with gauze following which the sockets were compressed. Post-extraction bleeding was determined to be significantly $(\mathrm{P}<0.01)$ controlled by PVP-I treatment as compared to saline. ${ }^{29}$ The anti-hemostatic effect may help patients with bleeding from severe mucositis and this needs to be investigated further.

From these findings, it appears that the benefits of PVP-I for the treatment of inflammatory conditions such as OM could be broader than its effects as an anti-microbial agent. The long track record of safe and efficacious use of PVP-I in clinical settings is also an advantage for further clinical investigation.

The current options available for OM management in clinical trials and clinical practice are severely limited. Keefe et al. has encouraged the pharmaceutical industry to refocus its efforts on developing such agents in areas of unmet need, including OM and chemotherapy-induced nausea/vomiting. ${ }^{30}$ The considerable unmet medical needs that remain for OM necessitate the investigation of a wider range of therapies.

The authors suggest that there is moderate scientific evidence for benefit provided by PVP-I in the management of OM; however, additional studies are encouraged. The authors also agree that based on the available evidence, PVP-I can be recommended for the prevention and treatment of OM.

\section{References}

1. Chaveli-López B, Bagán-Sebastián JV. Treatment of oral mucositis due to chemotherapy. J Clin Exp Dent 2016;8:e2019.

2. Bensinger W, Schubert M, Ang KK, et al. NCCN task force report: prevention and management of mucositis in cancer care. JNCCN 2008;6;Suppl 1.

3. Raber-Durlacher J. Current practices for management of oral mucositis in cancer patients. Support Care Cancer 1999;7:71.

4. Negrin RS, Toljanic AJ. Oral toxicity associated with chemotherapy. Available from: http://www.uptodate.com/contents/oral-toxicity-associated-with-chemotherapy Accessed: Oct 7, 2016.

5. Sonis ST. Efficacy of palifermin (keratinocyte growth factor-1) in the amelioration of oral mucositis. Core Evid 2010;4:199-205.

6. Wang HM, Lin CY, Hsieh CH, et al. Induction chemotherapy with dose-modified docetaxel, cisplatin, and 5-fluorouracil in Asian patients with borderline resectable or unresectable head and neck cancer. J Formos Med Assoc 2016;16:30015-8.

7. Dodd MJ, Dibble SL, Miaskowski C, et al. Randomized clinical trial of the effectiveness of 3 commonly used mouthwashes to treat chemotherapy-induced mucositis. Oral Surg Oral Med Oral Pathol Oral Radiol Endod 2000;90:39-47.

8. El-Housseiny AA, Saleh SM, El-Masry AA, Allam AA. The effectiveness of vitamin ' $E$ ' in the treatment of oral mucositis in children receiving chemotherapy. J Clin Pediatr Dent 2007;31:167-70.

9. Bowen JM, Elad S, Hutchins D, Lalla RV. Methodology for the MASCC/ISOO mucositis clinical practice guidelines update. Support Care Cancer 2013;21:303-8.

10. Lalla RV, Bowen J, Barasch A, et al. MASCC/ISOO clinical practice guidelines for the management of mucositis secondary to cancer therapy. Cancer 2014;120:1453-61. 
11. Lachapelle J-M, Castel O, Fueyo Casado A. Antiseptics in the era of bacterial resistance: a focus on povidone iodine. Future Med 2013;10:579-92.

12. Leaper DJ, Durani P. Topical antimicrobial therapy of chronic wounds healing by secondary intention using iodine products. Int Wound J 2008;5:361-8.

13. Kanagalingam J, Feliciano R, Hah JH, et al. Practical use of povidone-iodine antiseptic in the maintenance of oral health and in the prevention and treatment of common oropharyngeal infections. Int J Clin Pract 2015;69:1247-56.

14. Cooper RA. Iodine Revisited. Int Wound J 2007;4:124-37.

15. Ripa S, Bruno R, Reder R. Clinical applications of povidoneiodine as a topical antimicrobial. Handbook of topical antimicrobials industrial applications industrial applications in consumer products and pharmaceuticals. Boca Raton, FL: CRC Press; 2002.

16. Konig B, Reimer K, Fleischer W, Konig W. Effects of Betaisodona on parameters of host defense. Dermatology 1997; 195:42-8.

17. Beukelman CJ, van den Berg AJ, Hoekstra MJ, et al. Antiinflammatory properties of a liposomal hydrogel with povidone-iodine (Repithel) for wound healing in vitro. Burns 2008;34:845-55.

18. Rahn R, Adamietzh LA, Boettcher HD, et al. Povidone-lodine to prevent mucositis in patients during antineoplastic radiochemotherapy. Dermatology 1997;195:57-61.

19. Madan PDK, Squeira PS, Shenoy K, Shetty J. Povidone-lodine to prevent mucositis in patients during antineoplastic radiochemotherapy. J Cancer Res Ther 2008;4:1.

20. Yoneda S, Imai S, Hanada N, et al. Effects of oral care on development of oral mucositis and microorganisms in patients with esophageal cancer. Jpn J Infect Dis 2007;60:23-8.

21. Roopashri G, Jayanthi K, Guruprasad R. Efficacy of benzydamine hydrochloride, chlorhexidine, and povidone iodine in the treatment of oral mucositis among patients undergoing radiotherapy in head and neck malignancies: a drug trail. Contemporary Clin Dentistry 2011;2:1.

22. Hejna M, Kostler WJ, Raderer M, et al. Decrease of duration and symptoms in chemotherapy-induced oral mucositis by topical GM-CSF: results of a prospective randomised trial. Eur J Cancer 2001;37:1994-2002.

23. Rao S, Dinkar C, Vaishnav LK, et al. The Indian spice turmeric delays and mitigates radiation-induced oral mucositis in patients undergoing treatment for head and neck cancer: an investigational study. Integrat Cancer Ther 2014;1:201-10.

24. Lalla RV, Brennan MT, Schubert MM. Oral complications of cancer therapy. In: Yagiela JA, Dowd FJ, Johnson BS, et al., eds. Pharmacology and therapeutics for dentistry, 6th ed. St. Louis, MO: Mosby Elsevier; 2011. pp 782-798.

25. Keefe DM, Schubert MM, Elting LS, et al. Updated clinical practice guidelines for the prevention and treatment of mucositis. Cancer 2007; 109:820-31.

26. König B, Reimer K, Fleischer W, König W. Effects of Betaisodona ${ }^{\circledR}$ on parameters of host defense. Dermatology 1997; 195:42-8.

27. European Wound Management Association (EWMA). Position Document, 2006. Available from: http://old.ewma.org/fileadmin/user_upload/EWMA/pdf/Position_Documents/2006/Engl ish_pos_doc_2006.pdf Accessed: Oct 10, 2016.

28. Lachapelle J-M, Castel O, Fueyo Casado A. Antiseptics in the era of bacterial resistance: a focus on povidone iodine. Future Med 2013;10:579-92.

29. Arakeri G, Brennan PA. Povidone-iodine: an anti-oedematous agent? Int J Oral Maxillofac Surg 2011;40:173-6.

30. Keefe D, Al Garni A, Villalon A, et al. Challenges in supportive cancer care: perspectives from the Asia Pacific and Middle East. Support Care Cancer 2016;24:4479-81. 